\title{
TOTAL REPLACEMENT FOR POST-TRAUMATIC ARTHRITIS OF THE ELBOW
}

\author{
BERNARD F. MORREY， ROBERT A. ADAMS， RICHARD S. BRYAN
}

From the Mayo Clinic, Rochester, Minnesota

\begin{abstract}
Fifty-three of 55 consecutive elbow replacements for post-traumatic arthritis were followed for a minimum of two years (mean 6.3, range 2 to 14.4). The patients presented difficult management problems, having undergone an average of two previous operations per joint; 22 joints had suffered prior complications; $18 \mathrm{had}$ less than $50^{\circ}$ of flexion and six were flail. One of three versions of the Coonrad prosthesis was employed in all.

During the follow-up period, 10 patients underwent 14 revision procedures for aseptic loosening; 38 elbows are currently without progressive radiolucent lines. In two patients an elbow had to be resected, one for deep infection and the other for bone resorption following a foreign-body reaction to titanium.

The current design of the Coonrad prosthesis offers a reliable option for the treatment of post-traumatic arthritis but should be used only in carefully selected patients over the age of 60 years.
\end{abstract}

The early failures of total elbow arthroplasty have been attributed to poor patient selection and inadequate prosthetic design. Alternative procedures for the posttraumatic stiff, painful or unstable elbow are few. Arthrodesis is not an attractive option since sound fusion is difficult to achieve and it gives rise to considerable functional impairment. Soft-tissue releases about stiff joints have had unpredictable results. Distraction arthroplasty shows promise for patients under the age of 60 whose main complaint is of limitation of motion rather than pain. However, this procedure has only been developed recently and was not an option during the period we report.

Total replacement has proved increasingly reliable for rheumatoid arthritis of the elbow (Davis et al 1982; Pritchard 1983; Ewald and Jacobs 1984; Morrey 1985; Bell, Gschwend and Steiger 1986; Ross, Sneath and Scales 1987) but previous reports of its use in the treatment of post-traumatic arthritis are discouraging (Morrey et al 1981; Ewald and Jacobs 1984). The functional demands of a patient with rheumatoid arthritis are less than those of a patient with post-traumatic

B. F. Morrey, MD, Professor and Chairman

R. A. Adams, OPA

R. S. Bryan, MD

Department of Orthopaedics, Mayo Clinic, 200 First Street, Rochester, Minnesota 55905, USA.

Correspondence should be sent to Dr B. F. Morrey.

(C) 1991 British Editorial Society of Bone and Joint Surgery 0301-620X/91/4108 \$2.00

J Bone Joint Surg [Br] 1991 ; 73-B : 607-12. arthritis, and the latter have often had several previous operations resulting in serious loss of bone.

No previous report has dealt exclusively with the treatment of post-traumatic arthritis. We have, therefore, reviewed our experience in treating this condition with three variants of a single prosthetic design.

\section{MATERIALS AND METHODS}

From 1973 to 1985 , 55 consecutive surgical procedures were performed on 54 patients using the original Coonrad implant or one of its two modifications (Coonrad 1979; Morrey 1985). There were 32 females and 22 males; one female had bilateral procedures. The average age of the group was 56.4 years (range, 22 to 80 ).

The implant. The original and the modified Coonrad designs were defined as follows: type I (1973 to 1978) was a titanium implant with a $10 \mathrm{~cm}$ stem and a constrained high density polyethylene hinge. This design was found to loosen at an unacceptable rate in rheumatoid patients (Morrey et al 1981). Biomechanical studies suggested that a loose or 'toggle' hinge which allowed some varus/ valgus laxity and some axial rotation would approximate more closely to the normal kinematics of the joint (Morrey and Chao 1978). Consequently, the type II implant (1979 to 1981) provided varus/valgus laxity of $7^{\circ}$ to $8^{\circ}$. Further studies of the pattern of failure, and additional biomechanical evidence, indicated that loosening was usually due to posterosuperior displacement of the implant due to the cyclic application of loads of nearly three times the body-weight (Morrey 1985). Hence, the present design (type III, used since 1981) has a type 
II 'loose hinge' and a $15 \mathrm{~cm}$ humeral stem with an anterior flange to resist posterosuperior displacement. The distal part of the humeral component and the proximal part of the ulnar component are coated with a titanium plasma spray for better cement fixation (Fig. 1). To enhance rotational fixation and to decrease stress at the bone-cement interface, a bone graft is placed between the anterior cortex of the humerus and the prosthetic flange. The articulation is locked by a split ring; this feature has remained unchanged through the last two implant types and has not failed to date.

In the 55 operations we used 19 type I, 10 type II, and 26 type III implants. The indications for surgery, the clinical features and the results are summarised, according to implant type, in Tables I to III.

Indications. The primary indication for surgery was pain $(76 \%)$. In six cases the operation was considered a salvage procedure because of severe bone loss and in seven the indication was an ankylosed joint or one with less than $20^{\circ}$ of motion. At the beginning of our series, elbow replacement was performed on some young patients but as experience showed that failure was associated with youth, we later employed a more discriminative approach. Because of this change in policy the average age of the patients who had type I and II implants was 51 years and the average age of those who had type III implants was 61 years.

Bone loss. The pre-operative pathology was graded according to the amount of distal humeral bone which had been destroyed at the time of injury or prior surgery. Bone loss was classified as follows: grade 1, articular surfaces damaged but trochlea and capitellum present; grade 2, absence of the trochlea but preservation of the medial and lateral supracondylar columns; grade 3, absence of either the medial or the lateral supracondylar column; grade 4, loss of the whole distal humerus up to or above the level of the olecranon fossa (Fig. 2).

Ulnar bone loss was classified according to the presence or absence of the olecranon. The significance of a deficient olecranon is the lack of an osseous attachment for the triceps.

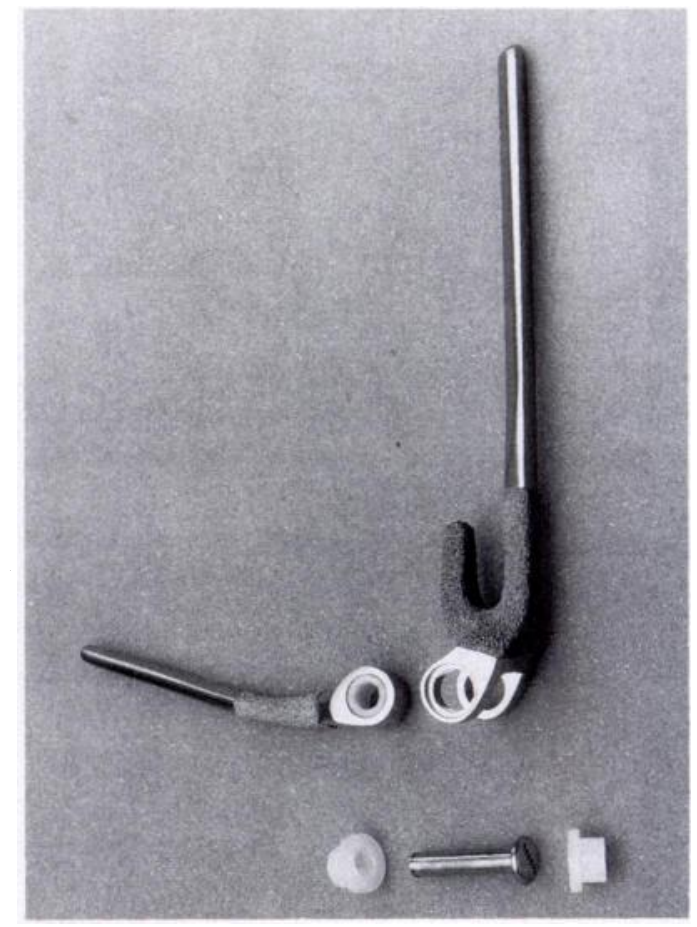

Fig. 1

The most recent prosthesis consists of a hinge articulation with $7^{\circ}$ of varus/valgus laxity, rough surfaces on the distal humeral and proximal ulnar components, and a flange which, when incorporated with bone grafts, resists posterior displacement and torsion of the humeral stem.

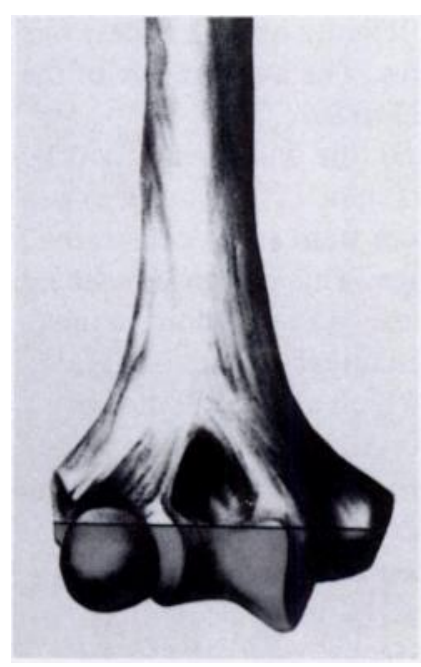

Fig. 2a

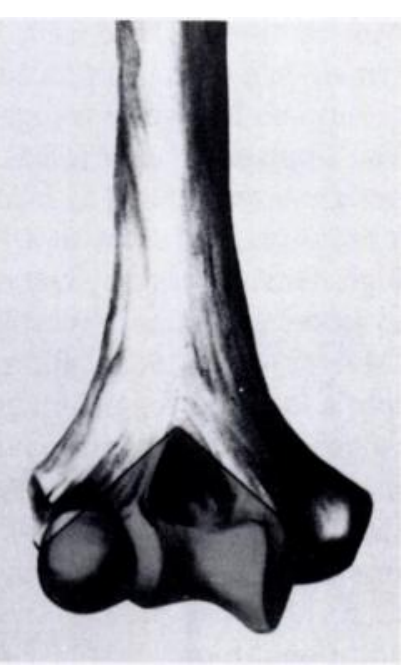

Fig. 2b

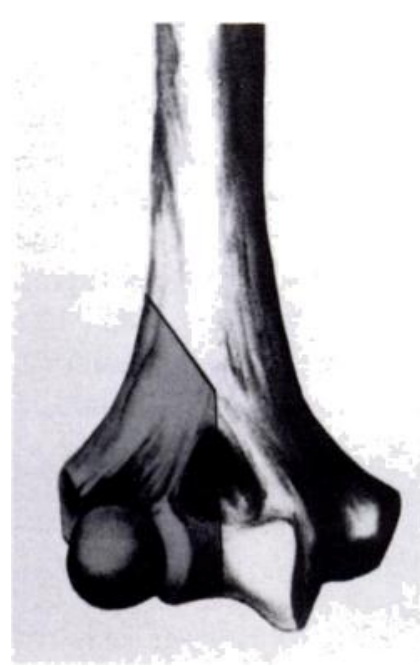

Fig. 2c

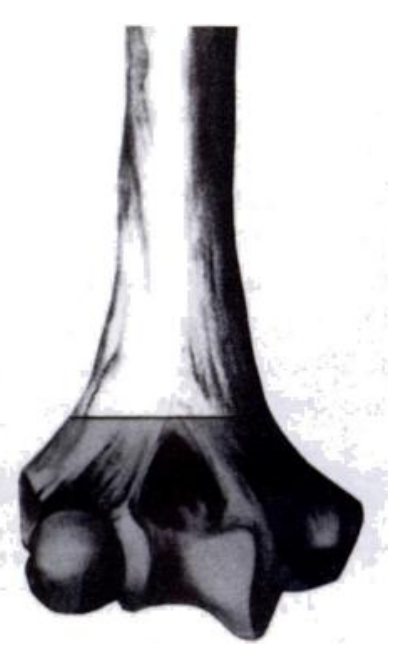

Fig. 2d

Classification of bone loss in the humerus. Figure $2 \mathrm{a}$ - grade 1 : some parts of the articular surfaces remain. Figure 2b - grade 2: only the supracondylar columns are preserved. Figure $2 \mathrm{c}$-grade 3: There is an ununited fracture of the medial or lateral condyle. Figure $2 \mathrm{~d}$-grade 4: loss of bone up to or above the olecranon fossa. 


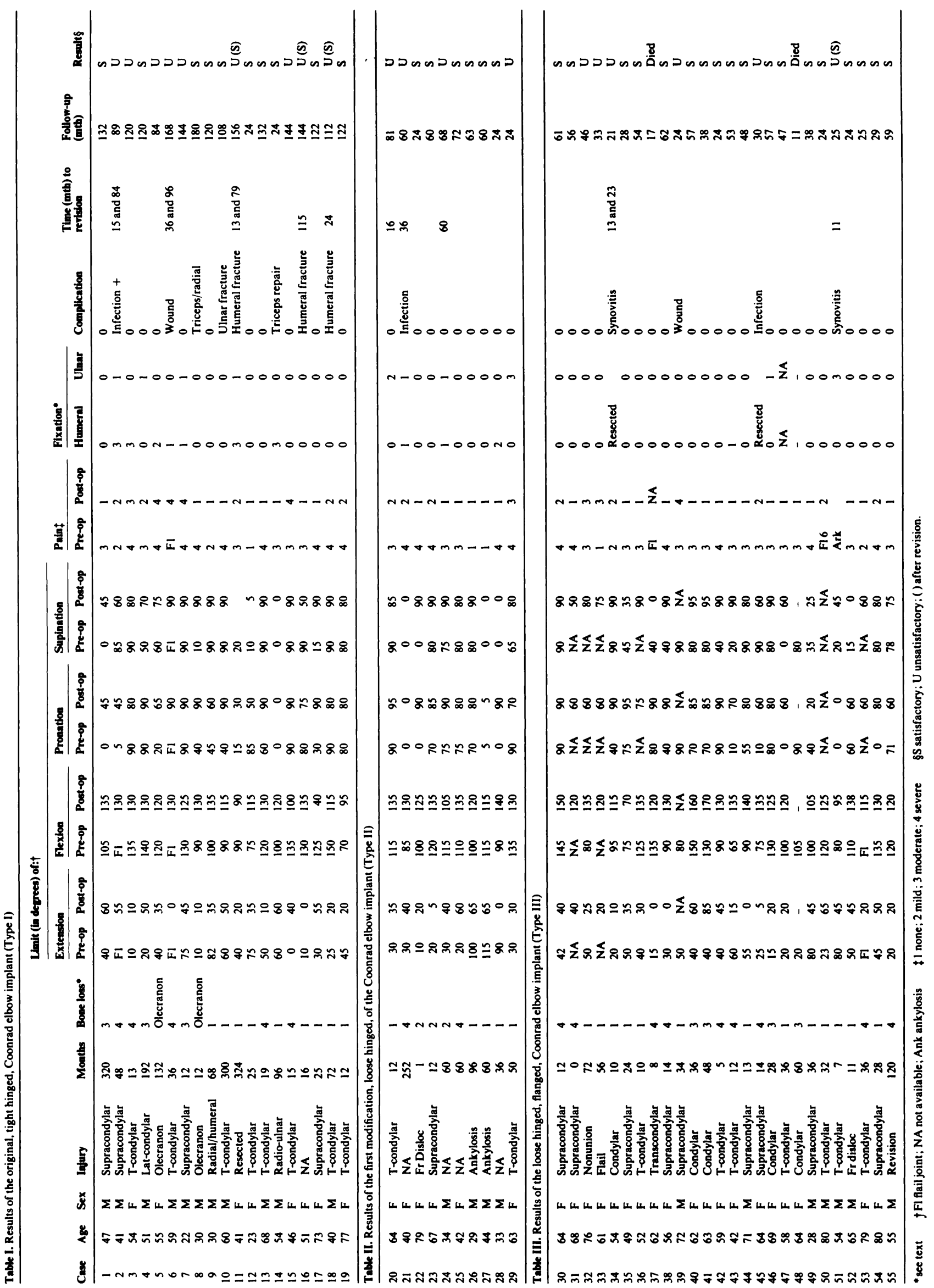


Prior surgery. The patients were further subdivided according to the number of prior surgical procedures and previous attempts at reconstruction. A mean of 1.8 previous surgical procedures had been performed per patient. Seven elbows had been previously treated by manipulation and immobilisation in a plaster cast; 21 had undergone a single open reduction and internal fixation; 24 had undergone from two to eight previous procedures. In addition to reduction and fixation of fractures, 11 elbows had undergone previous attempts at reconstruction. These included eight total replacements and three resection or interposition arthroplasties (see Fig. 2d). Six had ended up with flail joints.

Prior complications. At the time of presentation to us, 11 patients had some degree of ulnar neuropathy and three had partial radial neuropathy, either as the result of the initial injury or from later surgery.

Follow-up. Fifty-three of the elbows were reviewed more than two years after arthroplasty; two patients had died of unrelated causes prior to this examination. Thirty-one elbows were examined by us and nine by other orthopaedic surgeons; 13 patients answered a questionnaire. Radiographic follow-up was available for all 53 elbows.

The mean radiographic follow-up was 5.6 years $(2$ to 14); clinical follow-up averaged 6.3 years (2 to 14.4). The lateral radiograph was used to assess the status of the bone-cement interface which was graded as follows: type 0 , a lucent line less than $1 \mathrm{~mm}$ thick and involving less than $50 \%$ of the interface; type I, a lucent line of $1 \mathrm{~mm}$ or more involving less than $50 \%$ of the interface; type II, more than $1 \mathrm{~mm}$ lucency involving more than $50 \%$ of the interface; type III, more than $2 \mathrm{~mm}$ of lucency around the entire interface; type IV, gross loosening (Fig. 3).

A satisfactory result means that the patient does not use analgesics, and is satisfied with the outcome, radiographs show no progressive lucency, and no subsequent revision operation had been required.

\section{RESULTS}

The results are recorded in tabular form under three headings according to the implant used (Tables I to III).

After the initial procedure, 34 of the 53 elbows were rated satisfactory (64\%). These included ten of the 19 type I implants; six of the 10 type II, and 18 of the 24 type III. After revision and re-implantation of those implants that had loosened, 38 of the 53 elbows were rated as satisfactory $(72 \%)$.

Movement. The mean range of flexion/extension before operation was $41^{\circ}$ to $111^{\circ}$. After joint replacement, the range was $34^{\circ}$ to $128^{\circ}$.

The mean ranges of pronation and supination before surgery were $58^{\circ}$ and $64^{\circ}$ respectively. After joint replacement, the ranges were $68^{\circ}$ and $76^{\circ}$. The range of movements obtained after surgery did not differ with the three designs of implant.
Complications. There were 11 instances of loosening of the implant. One was due to infection and two to foreignbody synovitis; eight had mechanical loosening. Ten patients had complications other than loosening, to give a total of $21(38 \%)$ with complications. The $62 \%$ complication rate, with the type I and II designs, decreased to $16 \%$ with the type III implant. This difference is statistically significant $(\mathrm{p}<0.001)$.

Loosening and revision. The failures, as related to the implant type, are shown in Tables I to III. In the 10 initial revisions for aseptic loosening the humeral component was loose in six (Fig. 3), both components were loose in three and one had loosening of the ulnar component.

Five of the 19 type I designs were revised for loosening at an average of 40.6 months after surgery. Of these, three have become loose a second time. Three of the 10 type II designs were revised for loosening at an average of 37.1 months after surgery.

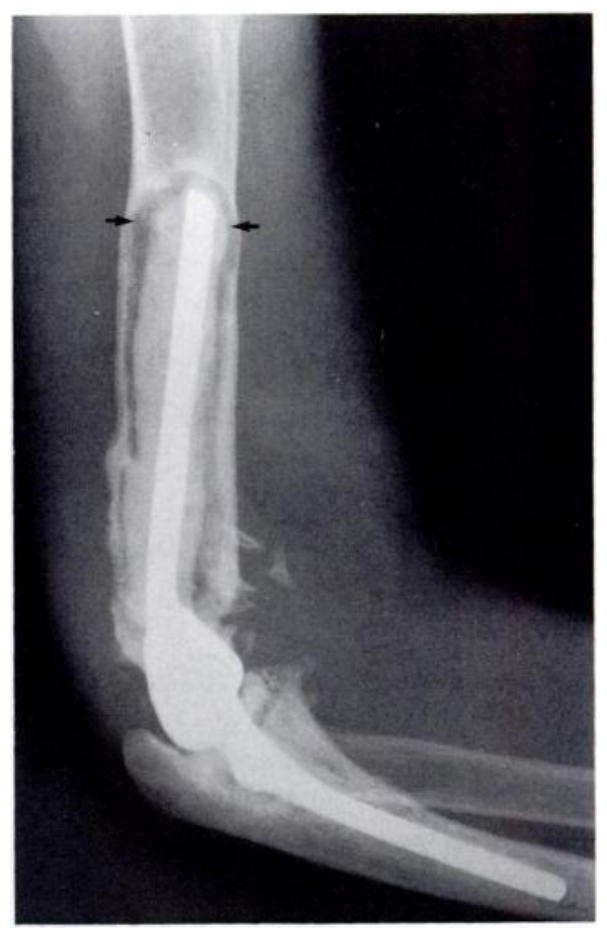

Fig. 3

Gross loosening with alteration of the bone and thinning of the cortex. A type I Coonrad prosthesis three years after implantation.

Two cases of non-mechanical aseptic loosening have occurred with the type III implant. The manufacturing process of the type III device employed a plasma spray which allowed for some shedding of particulate titanium. Small particles migrated into the bushing interface causing the titanium to become scored and to produce further particulate debris. This gave rise to a foreign- 


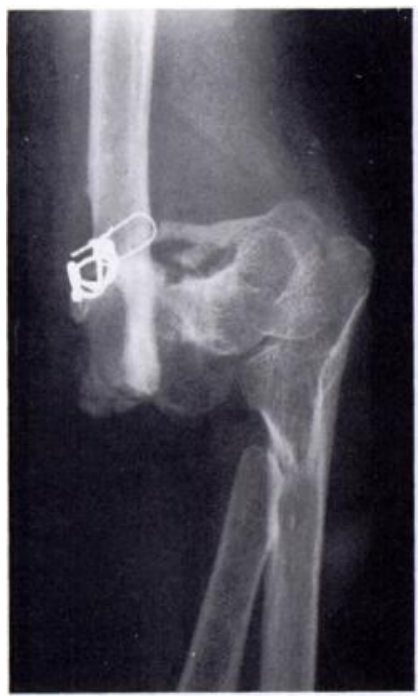

Fig. 4a

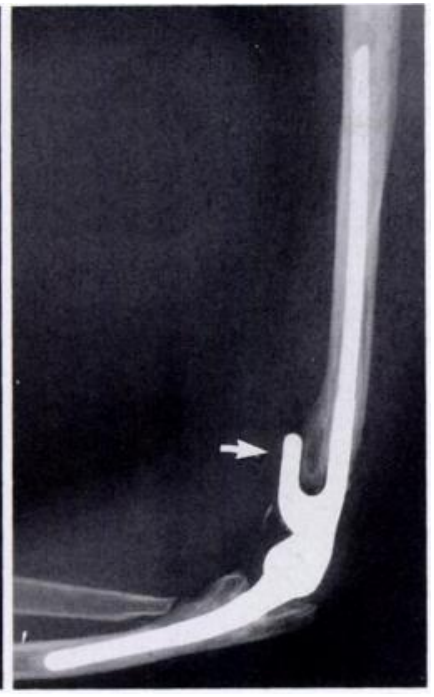

Fig. 4b
Figure 4a - An ununited supracondylar fracture in a 67-year-old woman. Figure $4 \mathrm{~b}-$ Five years after arthroplasty with a type III implant there is an intact bone-cement interface and incorporation of the bone graft behind the flange (arrow).

body reaction which caused the implant to loosen. These two cases were revised at 11 and 13 months. One of these became loose again and the second implant was removed. Another patient with a type III device required revision for deep infection. In the remainder the implants appear to be secure (Figs 4 and 5) except for one case in which there is a non-progressive lucent line.

Radiographic lucency. The results of the analysis of the most recent radiographs are shown in Tables I to III.

In the type I device there was a greater incidence of lucency about the humeral than about the ulnar compo- nent. In the less constrained implants (type II and III), there was a lower incidence of humeral lucencies.

\section{DISCUSSION}

Recent improvements in prosthetic design and surgical technique have resulted in more reliable elbow replacements for patients with rheumatoid arthritis (Morrey et al 1981; Davis et al 1982; Ewald and Jacobs 1984; Bell et al 1986). However, joint replacement in post-traumatic arthritis has had a far greater failure rate (Inglis and Pellicci 1980; Davis et al 1982; Ewald and Jacobs 1984; Lowe et al 1984). These patients are relatively young and, since there are no other joints involved, they make greater demands on the prosthesis.

Post-traumatic arthritis is often quite disabling due to pain and loss of motion; bone deficiency is common and a flail lim $\bar{b}$ may result from nonunion of a fracture. Of our patients $29 \%$ had complete absence and $13 \%$ partial absence of the distal humerus. There was severe loss of movement in $13 \%$; another $11 \%$ had a substantially functionless joint due to gross instability. Such circumstances represent a relative contra-indication to most designs of joint replacement (Coonrad 1979).

Unfortunately, there is no reliable alternative treatment. Arthrodesis is not a good salvage procedure since the essential function of the elbow is to allow the hand to be placed in space by flexion and extension of the joint. Interposition arthroplasty, the traditional treatment for these conditions, has unpredictable results and allograft replacement has as high a complication rate as prosthetic replacement, with added risks of late deterioration (Urbaniak and Black 1985). Prosthetic replacement, especially in the older age group, remains the best treatment.

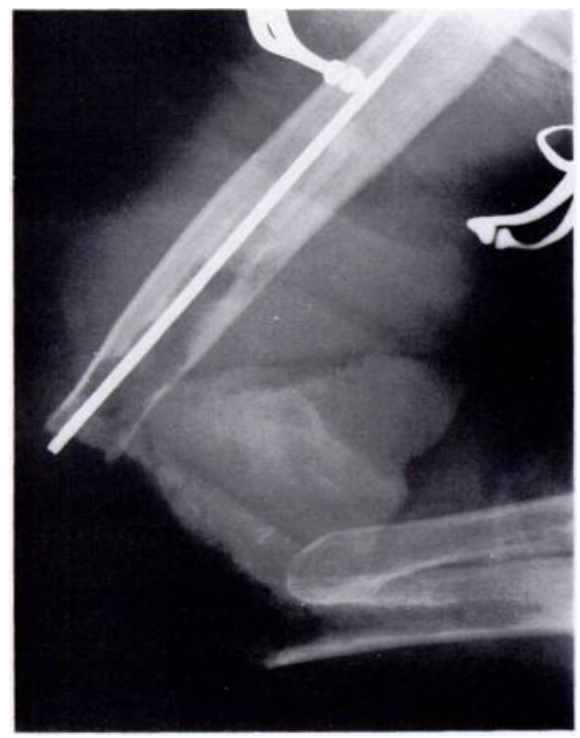

Fig. 5a

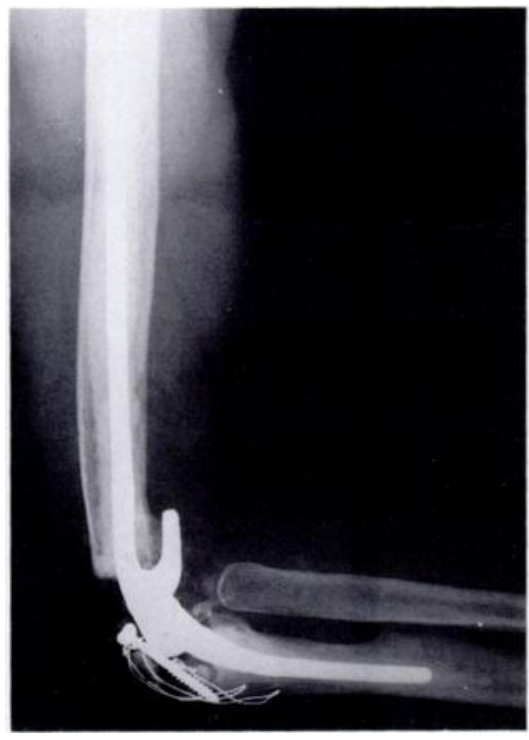

Fig. 5b
Figure 5a - Intra-operative radiograph after removal of a loose elbow prosthesis. Figure $5 b$ - Three years after its replacement with a modified Coonrad implant (type III). 
The poor results of our first implants had led us to advise against elbow arthroplasty in post-traumatic arthritis (Morrey et al 1981). However, some things have changed since our earlier operations. Improved surgical exposure by the Mayo approach (Bryan and Morrey 1982) has diminished the number of intra-operative fractures; injection of cement produces a better bonecement interface and a 'loose' hinge decreases the forces that are transmitted across that interface.

Apart from the design changes noted above, patient selection has become more discriminatory. The mean age of treated patients has risen from 48.5 years in the group with type I to 61.8 in those with type III. Of the patients with types I and II, 34\% had significant loss of bone from the distal humerus compared to $50 \%$ of those given type III implants. We now impose strict limitations on the use of the limb; heavy work is not allowed and a lifting limit of $10 \mathrm{lb}(4.5 \mathrm{~kg})$ is recommended.

The type III implant, followed up for an average 3.2 years has proved effective for the treatment of posttraumatic arthritis in older patients. The flanged shape of the humeral component has proved secure even in cases with severe bone loss. Custom-made prostheses have not been required since the type III design was introduced in 1981. This is a distinct advantage compared to the need to fabricate special prostheses for patients with post-traumatic arthritis (Figgie, Inglis and Mow 1986; Ross et al 1987). The complication caused by a foreign-body reaction to particulate titanium has not been observed since 1985 when the manufacturing process was changed. The current design is coated with titanium beads instead of a plasma spray and has given no evidence of particulate synovitis.

Despite our favourable results with the recent type III design we do not advocate prosthetic replacement for post-traumatic arthritis in individuals under 60 years of age. Total elbow arthroplasty should only be considered as the last option in such cases. Resection and distraction arthroplasty, which has produced gratifying results in recent years, may be a better procedure. Nonunion of a fracture of the distal humerus in younger patients should be treated by conventional methods of osteosynthesis. However, acceptable and reliable results can be achieved by elbow arthroplasty. Overall, we have achieved a functioning and painless elbow in $74 \%$ of these difficult cases over the last 13 years.

One or more of the authors have received or will receive benefits for personal or professional use from a commercial party related directly or indirectly to the subject of this article. In addition benefits have also been or will be directed to a research fund, foundation, educational institution, or other non-profit institution with which one or more of the authors is associated.

\section{REFERENCES}

Bell S, Gschwend N, Steiger U. Arthroplasty of the elbow : experience with the Mark III GSB prosthesis. Aust NZ J Surg 1986; 56 : 823-7.

Bryan RS, Morrey BF. Extensive posterior exposure of the elbow: a triceps-sparing approach. Clin Orthop 1982; 166:188-92.

Coonrad RW. History of total elbow arthroplasty. In: Inglis AE, ed. Symposium on total joint replacement of the upper extremity. Chicago AAOS, 1979:75-90.

Davis RF, Weiland AJ, Hungerford DS, Moore JR, Volenec-Dowling S. Nonconstrained total elbow arthroplasty. Clin Orthop 1982; $171: 156-60$.

Ewald FC, Jacobs MA. Total elbow arthroplasty. Clin Orthop 1984; $182: 137-42$.

Figgie HE III, Inglis AE, Mow C. Total elbow arthroplasty in the face of significant bone stock or soft tissue losses: preliminary results of custom-fit arthroplasty. J Arthroplasty 1986; 1(2):71-81.

Inglis AE, Pellicei PM. Total elbow replacement. J Bone Joint Surg [Am] 1980; 62-A:1252-8.

Lowe LW, Miller AJ, Allum RL, Higginson DW. The development of an unconstrained elbow arthroplasty: a clinical review. $J$ Bone Joint Surg [Br] 1984; 66-B :243-7.

Morrey BF. The elbow and its disorders. WB Saunders, 1985.

Morrey BF, Chao EYS. Passive motion of the elbow joint. J Bone Joint Surg [Am] 1976; 58-A :501-8.

Morrey BF, Bryan RS, Dobyns JH, Linscheid RL. Total elbow arthroplasty: a five-year experience at the Mayo Clinic. J Bone Joint Surg [Am] 1981 ; 63-A :1050-63.

Pritchard RW. Anatomic surface elbow arthroplasty: a preliminary report. Clin Orthop 1983; 179:223-30.

Ross AC, Sneath RS, Scales JT. Endoprosthetic replacement of the humerus and elbow joint. $J$ Bone Joint Surg $[B r] 1987 ; 69-B$ : 652-5.

Urbaniak JR, Black KE Jr. Cadaveric elbow allografts: a six-year experience. Clin Orthop 1985; 197:131-40. 